\title{
MAGNETIC DESIGN OF A HIGH GRADIENT QUADRUPOLE FOR THE LHC LOW- $\beta$ INSERTIONS
}

\author{
G. Sabbi, S.A. Gourlay, J. Kerby, M.J. Lamm, P.J. Limon, F. Nobrega, I. Novitski, \\ J.B. Strait, A.V. Zlobin, FNAL ${ }^{1}$, S. Caspi, R. Scanlan, LBNL ${ }^{1}$, A. Ghosh, R. Gupta, BNL ${ }^{1}$
}

\section{Abstract}

Fermilab, Lawrence Berkeley National Laboratory and Brookhaven National Laboratory have formed a consortium to provide components for the Large Hadron Collider (LHC) to be built at CERN. The U.S. contribution includes half of the high gradient quadrupoles (HGQ) for the inner focusing triplets. In this paper a description of the HGQ magnetic design is given, including short sample limit for field gradient, sources and expected values of systematic and random field errors, and possible strategies for field quality correction.

\section{INTRODUCTION}

In order to achieve a luminosity in excess of $10^{34} \mathrm{~cm}^{-2} \mathrm{~s}^{-1}$ at the LHC, special high gradient quadrupoles are required for the final focusing triplets [1]. These magnets must provide a field gradient of $235 \mathrm{~T} / \mathrm{m}$ over a $70 \mathrm{~mm}$ bore, with a sufficient margin in order to withstand the heavy heat load due to secondary particles from beam-beam collisions. At the same time, due to large and rapidly varying values of the $\beta$-function, a high field quality is required. To meet these severe constraints, a design based on a two-layer coil geometry has been proposed [2]. The magnet uses SSCtype NbTi superconducting strands and operates in superfluid helium. The first short model of this design is being fabricated and will be tested this fall. In this paper, the magnetic design for the HGQ straight section is discussed.

\section{MAGNET DESIGN}

The HGQ design is based on four two-layer coils connected in series, surrounded by collar and yoke laminations. A thick-wall stainless steel beam tube also serves as an internal beam absorber. With respect to the last status report [2], the coil prestress is now entirely provided by the collars. For this purpose the collar material has been changed from aluminum to stainless steel, and the collar width at the midplane has been increased from 20 to $25 \mathrm{~mm}$. The yoke inner radius at the midplane is now $92.56 \mathrm{~mm}$ while the outer radius remains unchanged at $200 \mathrm{~mm}$. The yoke lamination features four round holes for the HeI heat exchanger and four rectangular holes for electrical connections, as well as special cutouts for magnet fabrication. In the pole regions, eight rectangular gaps between collars and yoke house tuning shims for field quality adjustment [3]. The resulting HGQ cross section is shown in Figure 1.

The cables for the magnet models are made of SSC NbTi strands with a critical current density of $2.75 \mathrm{kA} / \mathrm{mm}^{2}$ at $5 \mathrm{~T}$

\footnotetext{
${ }^{1}$ Supported by the U.S. Department of Energy.
}

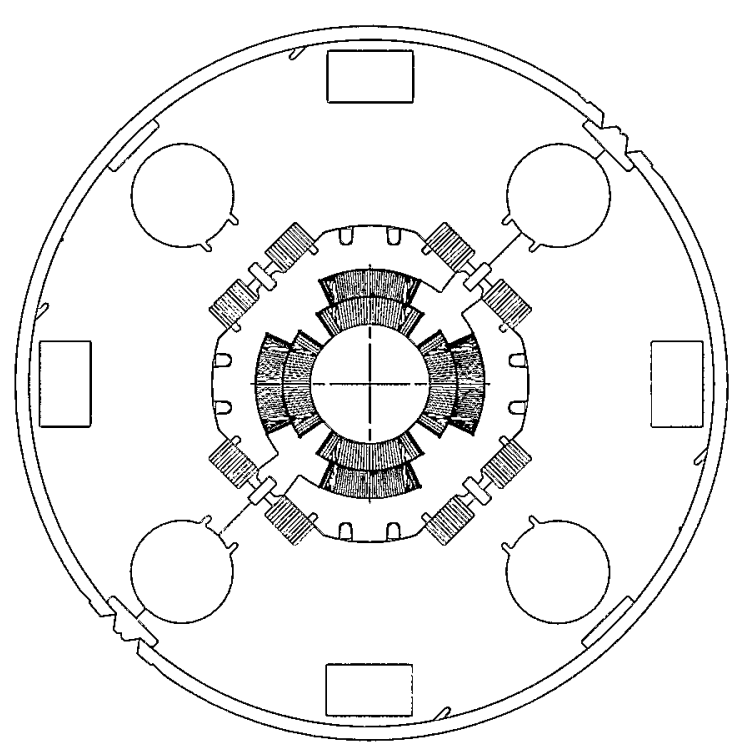

Figure 1: HGQ cross-section

and $4.2 \mathrm{~K}$. The SSC strands will eventually be replaced with improved ones that are now under development, with the goal of increasing the critical current density to $3.4 \mathrm{kA} / \mathrm{mm}^{2}$ at $5 \mathrm{~T}$ and $4.2 \mathrm{~K}$. Table 1 shows the HGQ short sample limits at $1.9 \mathrm{~K}$ operating temperature, calculated for both existing and new strands.

Table 1: Short sample limits.

\begin{tabular}{|c|c|c|c|c|}
\hline$J_{c}(5 \mathrm{~T}, 4.2 \mathrm{~K})$ & $G_{s s}$ & $I_{s s}$ & $B_{\max }^{(\text {inner })}$ & $B_{\max }^{\text {(outer) }}$ \\
$\mathrm{kA} / \mathrm{mm}^{2}$ & $\mathrm{~T} / \mathrm{m}$ & $\mathrm{kA}$ & $\mathrm{T}$ & $\mathrm{T}$ \\
\hline 2.75 & 253.8 & 14.2 & 9.9 & 8.2 \\
3.4 & 270.2 & 15.2 & 10.4 & 8.8 \\
\hline
\end{tabular}

The operating current is $0.8 \mathrm{kA}$ at injection and $13.1 \mathrm{kA}$ at nominal gradient. At nominal current, the magnet will operate at $93 \%$ along the load line with the SSC conductor and at $87 \%$ with the improved one.

\section{FLELD QUALITY ANALYSIS}

The design low-order harmonics for the HGQ central field, resulting from the cross-section optimization with a circular yoke of infinite permeability, are $b_{6}=-2 \cdot 10^{-3}$, $b_{10}=-1 \cdot 3 \cdot 10^{-5}$. All other normal and skew harmonics with $\mathrm{n}<14$ are zero. The harmonics here and below are given in units of $10^{-4}$ of the main quadrupole field at $1 \mathrm{~cm}$ reference radius. 


\subsection{Field error sources}

Mechanical effects. Due to insufficient keystoning of the cables, the coil curing mandrels do not provide a perfect radial constraint for some of the turns. It is expected that after curing the turns will be aligned to the outer mold radius. The validity of this assumption will be checked as part of the model magnet program. The uncertainty range corresponds to alignment of all turns to the inner mandrel radius.

The collared coil deformation during cooling down and springback under nominal prestress, as well as its deformation under Lorentz forces with respect to the warm unstressed geometry produce additional field errors. The Lorentz force effect is negligible at injection and maximal at collision.

Deviations from nominal prestress and turn/coil block displacements due to manufacturing tolerances contribute to the random errors.

Magnetization effects. The coil magnetization is determined by the critical current density in the superconductor, filament diameter, $\mathrm{Cu} / \mathrm{Sc}$ ratio, etc. This effect is maximal at injection and becomes negligible at collision. The uncertainty is related to the accuracy of the model and the parameters of the conductor under development.

The magnetization of the stainless steel collars and beam absorber produces current independent harmonics which depend on geometry and magnetic permeability. A collar permeability of 1.003 is expected. However, depending on the exact chemical composition and the heat treatment, larger values up to 1.006 may be obtained. A beam absorber with $27 \mathrm{~mm}$ inner radius, $6 \mathrm{~mm}$ thickness and magnetic permeability 1.005 has been analyzed. Both the systematic effect and the random contribution due to misalignment were found to be negligible if the offset is below $0.5 \mathrm{~mm}$.

The yoke cross section was optimized to minimize the change in the harmonics due to iron saturation and to compensate the harmonics due to collar magnetization. The uncertainty is related to a precise knowledge of the iron $\mathrm{B}-\mathrm{H}$ characteristic.

Iron is the material of choice for the cryostat vacuum vessel. The cold mass is placed off-center with respect to this shell. The effect on the harmonics has been analyzed and found to be negligible.

Magnetic measurements. The field quality of each HGQ magnet will be determined during magnetic measurements. Measurement errors contribute to the uncertainty in the systematic harmonics and to the harmonic RMS spread. Since the measurement system is still being designed, the estimates have been done based on data available in $[4,5]$.

\subsection{Systematic effects}

Table 2 shows the main contributions to the $b_{6}$ harmonic component. Since all the contributions represent small perturbations to the main field, they are treated as independent effects. The total value of $b_{6}$ is $-0.09[-0.14,+0.04]$ at injection and $+0.01[-0.14,+0.02]$ at collision. For the $b_{10}$ component the same approach has been used and the following
Table 2: Systematic $b_{6}$.

\begin{tabular}{|l|r|c|}
\hline \multicolumn{1}{|c|}{ Effect } & Expected & Uncertainty \\
\hline Coil optimization & -0.002 & \\
Turn alignment & 0.000 & $-0.134,+0.0$ \\
Prestress at 1.9 K & 0.027 & \\
Lorentz forces & -0.017 & \\
Coil magnetization & -0.11 & \pm 0.03 \\
Collar magnetization & -0.018 & $-0.018,+0.0$ \\
Yoke geometry & 0.016 & \\
Iron saturation & 0.005 & \pm 0.001 \\
Measurements & 0.000 & \pm 0.02 \\
\hline
\end{tabular}

values have been obtained: $+0.0001[-0.0011,+0.0009]$ at injection and $+0.0003[-0.0011,+0.0009]$ at collision.

Table 3 shows the expected uncertainty in the systematic errors resulting from the accuracy of magnetic measurements.

Table 3: $a_{n}, b_{n}$ measurement uncertainty.

\begin{tabular}{|l|c|}
\hline$n$ & $a_{n}, b_{n}$ uncertainty $( \pm)$ \\
\hline 3 & 0.16 \\
4 & 0.085 \\
5 & 0.042 \\
6 & 0.020 \\
7 & 0.0095 \\
8 & 0.0044 \\
9 & 0.0020 \\
10 & 0.00087 \\
\hline
\end{tabular}

\subsection{Random effects}

The RMS spread of geometrical harmonics due to manufacturing tolerances, prestress changes and radial alignment, as well as the RMS spread which has been assumed for the magnetic measurements are reported in Table 4.

Table 4: HGQ random errors (RMS spread).

\begin{tabular}{|r|l|l|l|}
\hline$n$ & \multicolumn{2}{|c|}{ Coil geometry } & \multicolumn{1}{c|}{ Measurement } \\
& \multicolumn{1}{|c|}{$b_{n}$} & $a_{n}$ & \multicolumn{1}{|c|}{$b_{n} \& a_{n}$} \\
\hline 3 & 0.97 & 0.96 & 0.080 \\
4 & 0.35 & 0.33 & 0.042 \\
5 & 0.089 & 0.090 & 0.021 \\
6 & 0.026 & 0.023 & 0.010 \\
7 & 0.0066 & 0.0067 & 0.0047 \\
8 & 0.0018 & 0.0016 & 0.0021 \\
9 & 0.00041 & 0.00040 & 0.00098 \\
10 & 0.00010 & 0.00010 & 0.00044 \\
\hline
\end{tabular}

As can be seen, the random errors are dominated by the geometrical component for $\mathrm{n}<8$, while for higher order harmonics the spread due to measurement errors dominates.

Figure 2 shows a comparison between the total estimated random errors for the HGQ normal harmonics and the results of measurements for the FNAL LBQ [4] and HERA 
arc quadrupoles [5]. Both magnets have a two-layer coil and a bore radius comparable to HGQ. The measured data have been rescaled to $1 / 3.5$ of the bore radius $(1.09 \mathrm{~cm}$ for LBQ and $1.07 \mathrm{~cm}$ for HERA quads). As can be seen, rather good agreement is found between calculated and measured values.

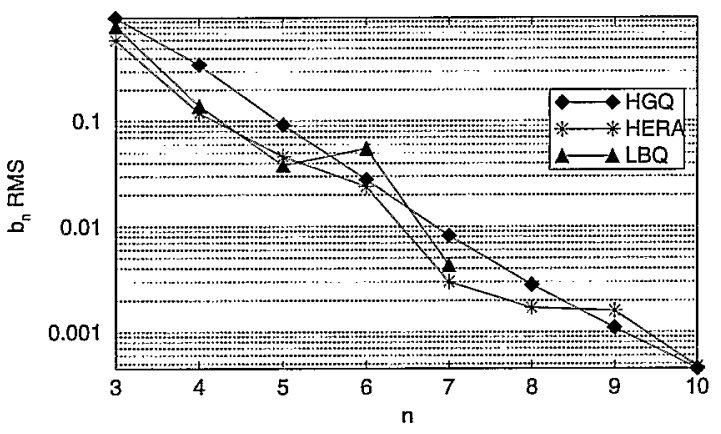

Figure 2: Random errors comparison (normal components).

\subsection{Field quality correction}

The correction scheme relies on modification of the coil geometry using pole and midplane shims in each octant, independently for the inner and outer layer. Larger corrections may be obtained by changing the thickness of the bearing strips, while for fine tuning of the harmonics insulation layers $25 \mu \mathrm{m}$ thick can be added or removed. The analysis shows that corrections of the systematic harmonics at nominal gradient within the predicted range of variation are possible using the existing tuning capability. Should larger effects appear, a cross-section iteration may be considered.

The RMS spread in the harmonics may be reduced either by coil reshimming after warm magnetic measurements or by the use of iron tuning shims. The first method provides a current-independent correction, but it is labor-intensive and subject to some uncertainty as the harmonics are not exactly reproducible after collared coil reassembling. The second method is very attractive in terms of required effort and reproducibility, but the resulting correction is currentdependent due to saturation effects. Preliminary calculations indicate that only $50 \%$ of the correction capability at low current is preserved at nominal gradient. The tuning shim correction scheme will be studied as part of the model magnet program.

\section{ERROR TABLES}

Based on field quality measurements carried out on the series of model magnets, correction schemes will be applied to make the average error at full excitation equal to zero. Some residual uncertainty applies due to the limitations of the correction scheme as well as the measurement accuracy. The harmonics at injection are then determined by the current dependent effects, with some additional uncertainty on $b_{6}$ related to the contribution from coil magnetization. The resulting systematic errors are listed in Table 5.
Table 5: Systematic errors.

\begin{tabular}{|r|l|l|}
\hline$n$ & $a_{n}, b_{n}$ mean & $a_{n}, b_{n}$ uncertainty $( \pm)$ \\
\hline 3 & 0.0 & 0.2 \\
4 & 0.0 & 0.09 \\
5 & 0.0 & 0.04 \\
6 & $0.0\left(-0.1^{*}\right)$ & $0.02\left(0.04^{*}\right)$ \\
7 & 0.0 & 0.01 \\
8 & 0.0 & 0.004 \\
9 & 0.0 & 0.002 \\
10 & $0.0\left(-0.0001^{*}\right)$ & 0.0009 \\
\hline
\end{tabular}

$\left(^{*}\right)$ data for $b_{n}$ at injection.

To generate the random error table, first the maximum between the estimated RMS spread of each HGQ harmonic component and the corresponding measured value from the LBQ and HERA quad productions was chosen. Then, for the low order non-allowed harmonics, and for all the higher order harmonics (where the measurement errors dominate), the normal and skew RMS errors have been made equal to the largest between the two. The resulting values are shown in Table 6.

Table 6: Random errors (RMS spread).

\begin{tabular}{|r|l|l|}
\hline$n$ & \multicolumn{1}{|c|}{$b_{n}$} & \multicolumn{1}{|c|}{$a_{n}$} \\
\hline 3 & 1.3 & 1.3 \\
4 & 0.4 & 0.4 \\
5 & 0.09 & 0.09 \\
6 & 0.06 & 0.03 \\
7 & 0.008 & 0.008 \\
8 & 0.003 & 0.003 \\
9 & 0.0016 & 0.0016 \\
10 & 0.0005 & 0.0005 \\
\hline
\end{tabular}

\section{SUMMARY}

The HGQ maximum gradient exceeds the LHC low- $\beta$ quadrupole design specification with a good margin. A detailed field quality analysis has been carried out, and estimates of the systematic and random field errors for the production magnets have been generated in a form suitable for dynamic aperture studies.

\section{REFERENCES}

[1] 'Large Hadron Collider Conceptual Design', CERN AC/9505 (LHC), 1995.

[2] R. Bossert et al., 'Development of a High Gradient Quadrupole for the LHC Interaction Regions', ASC '96, Pittsburgh, August 1996.

[3] R. Gupta et al., 'Tuning Shims for High Field Quality in Superconducting Magnets', MT-14, Finland, June 1995.

[4] R. Hanft et al., 'Magnetic Performance of New Fermilab High Gradient Quadrupoles', IEEE Particle Accelerator Conference (PAC91), S. Francisco, May 1991.

[5] J. Perot, J. M. Rifflet, 'Measurement Data Taken During the Industrial Fabrication of the HERA Superconducting Quadrupoles', Supercollider 3, Plenum Press, NY, 1991. 